\title{
Metrology for Monitoring of Endocrine Disrupting Chemicals Under the EU Water Framework Directive
}

\author{
LORIN STEINHAEUSER ${ }^{1}$, SOPHIE LARDY-FONTAN ${ }^{2}$, \\ CHRISTIAN PIECHOTTA ${ }^{1}$, ESTER HEATH ${ }^{3}$, STEFANIA \\ BALZAMO $^{4}$, CAROLINE GARDIA-PARÈGE $^{5}$, TANER \\ GÖKCEN $^{5}$, HELENE BUDZINSKI ${ }^{6}$ AND NOORA \\ PERKOLA $^{7}$ \\ ${ }^{1}$ Bundesanstalt für Materialforschung und -prüfung \\ ${ }^{2} \mathrm{LNE}$ \\ ${ }^{3} \mathrm{JSI}$ \\ ${ }^{4}$ ISPRA \\ ${ }^{5}$ TUBITAK UME \\ ${ }^{6}$ UMR CNRS 5805 EPOC \\ ${ }^{7}$ SYKE \\ Presenting Author: lorin.steinhaeuser@bam.de
}

Monitoring programs should generate high-quality data on the concentrations of substances and other pollutants in the aquatic environment to enable reliable risk assessment. Furthermore, the need for comparability over space and time is critical for analysis of trends and evaluation of restoration of natural environment. Additionally, research work and exercises at the European level have highlighted that reliable measurements of estrogenic substances at the PNEC level are still challenging to achieve.

The project EDC-WFD Metrology for monitoring endocrine disrupting compounds under the EU Water Framework Directive aims to develop traceable analytical methods for determining endocrine disrupting compounds and their effects, with a specific focus on three estrogens of the first watch list (17-beta-estradiol (17ßE2), 17-alpha-ethinylestradiol (EE2), and estrone (E1)). Environmental quality standards (EQS) for these compounds are at the ultra-trace level $(\mathrm{EQS} \mathrm{EE} 2=35 \mathrm{pg} / \mathrm{L}, \mathrm{EQS} \mathrm{E} 2=400 \mathrm{pg} / \mathrm{L}$, EQS E1 $=3600 \mathrm{pg} / \mathrm{L}$ ) and pose a significant challenge to analytical methods. Estrogens 17-alpha-estradiol $(17 \alpha \mathrm{E} 2)$ and estriol (E3) will be included to demonstrate the reliability of the developed methods and to support the requirements of Directive 2013/39/EC, Directive 2009/90/EC and Commission Implementation Decision (EU) 2018/840, hence improving the comparability and compatibility of measurement results within Europe. During the EDC-WFD project four selected effect-based methods (EBM) will be deeply investigated in order to improve their rationale use and their support in water quality assessment. In particular, the EBM sensitivity, specificity and accuracy on reference materials with single or mixture solutions of the five substances at a concentration of EQS values will be explored.

This contribution will present the objectives and methods applied within the EDC-WFD project.

Funding: This project (18NMR01) has received funding from the EMPIR programme co-financed by the Participating States and from the European Union's Horizon 2020 research and innovation programme. 